\title{
ARTÍCULO ORIGINAL - ORIGINAL ARTICLE \\ Ofidismo en el Paraguay: distribución geográfica, alteraciones fisiopatólogicas y necesidad de la preparación de un veneno de referencia nacional
}

\section{Ophidism in Paraguay: geographic distribution, physiopathological alterations, and the need for preparing a national reference venom}

Adolfo Borges ${ }^{1}$

${ }^{1}$ Centro para el Desarrollo de la Investigación Científica. Asunción, Paraguay.

Autor de correspondencia: borges.adolfo@gmail.com DOI: https://doi.org/10.32480/rscp.2019-24-2.249-261

Recibido: 10/10/2019. Aceptado: 25/11/2019.

Resumen: El accidente ofídico en el Paraguay es un evento frecuente que reviste gravedad si la serpiente asociada pertenece a las familias Viperidae y Elapidae, particularmente en los departamentos fronterizos con el Brasil, en donde afecta principalmente a hombres en actividades agrícolas entre los 20 y 40 años de edad. El oriente del Paraguay, junto con el sudeste del Brasil, ha sido identificado como una de las áreas de mayor incidencia de ofidismo en todo el Neotrópico. Se presenta una revisión de la problemática desde el punto de vista epidemiológico y fisiopatológico, conjuntamente con la distribución de las especies de potencial importancia médica de los géneros Bothrops y Micrurus. Se plantea la necesidad de elaborar un veneno de referencia nacional que represente las especies asociadas a los envenenamientos severos y que permita la evaluación de la capacidad neutralizante de antídotos foráneos, así como la eventual preparación de un antiveneno nacional.

Palabras clave: Bothrops, Micrurus, ofidismo, Paraguay, serpientes.

Abstract: Envenomation by poisonous snakes is frequent in Paraguay, particularly in the departments bordering with Brazil, and can be severe if the species involved belongs to the families Viperidae or Elapidae. It mostly affects 20-40 year old men involved in agricultural activities. Eastern Paraguay, together with southeast Brazil, has been identified as one of the highest snakebite incidence areas in the Neotropics. This review presents available epidemiological data and revises the physiopathology of the 
envenomation, particularly in the case of viperid venoms, as well as the current distribution of species in genera Bothrops and Micrurus in Paraguay. It posits the need to prepare a national reference venom based on Paraguay's biodiversity to pave the way for testing the neutralization capacity of foreign antivenoms or for the preparation of a national snake antivenom.

Keywords: Bothrops, Micrurus, ophidism, Paraguay, snakes.

\section{EPIDEMIOLOGÍA DEL ACCIDENTE OFÍDICO EN EL PARAGUAY}

El envenenamiento por serpientes (accidente conocido como ofidismo), es un problema de salud pública en toda la América Tropical, incluyendo Paraguay, que afecta principalmente a la población rural. Para el año 2004 se registraron en Paraguay 357 casos de ofidismo, con una tasa de incidencia de 6,2 casos por 100.000 habitantes, con una tasa de letalidad de 1,8\% (1), y para 2007 la cifra aumentó a 470 casos (2). La población en riesgo de envenenamiento por serpientes tóxicas en Paraguay está constituida por hombres jóvenes, entre 20 y 40 años de edad, agricultores y jornaleros, que suelen ser mordidos en las extremidades inferiores entre las 9:00-12:00H ó 17:00-20:00H (1). Los departamentos identificados como los de mayor prevalencia de ofidismo en 2004 han sido Concepción, Ñeembucú, Paraguarí, Guairá y Presidente Hayes (1). En 2015 fueron notificados 169 casos de accidente ofídico al Programa Nacional de Control de Zoonosis y Centro Antirrábico Nacional, siendo los departamentos identificados como de mayor prevalencia San Pedro, Itapúa y Caazapá (3). En un estudio posterior (4), que analizó datos nacionales entre 2010 y 2015, se pudo identificar que los departamentos de Alto Paraguay, Concepción, Amambay y Alto Paraná presentaron tasas de incidencia de ofidismo superiores a 15 casos por 100.000 habitantes (Figura 1). Aunque el ofidismo es un evento de denuncia obligatoria en el Paraguay (5), las estadísticas publicadas por lo general provienen de estudios realizados en centros de atención locales, por lo que es necesario el análisis de datos georreferenciados a nivel nacional que combinen la incidencia con la abundancia de las especies de potencial importancia médica, a fin de conocer la real dimensión espacial y temporal del problema similarmente a como se ha realizado en Costa Rica y Nicaragua (6). La tendencia, sin embargo, parece indicar que los departamentos fronterizos con el Brasil presentan la mayor prevalencia, correspondiendo éstos a zonas de alta densidad de especies del género Bothrops (7). El oriente del Paraguay y el sudeste del Brasil fueron identificadas como una de las áreas

Borges A. Ofidismo en el Paraguay: distribución geográfica, alteraciones fisiopatólogicas y necesidad de la preparación de un veneno de referencia nacional 
de mayor incidencia de ofidismo en todo el Neotrópico (8). El ofidismo es un problema sujeto a un potencial incremento epidemiológico debido a cambios en la dinámica de poblaciones de animales venenosos producto del cambio climático, como ha sido documentado para el norte de Argentina (9). Especies de Bothrops de importancia médica se han adaptado a los bosques secundarios producidos por la deforestación en varios países de América Latina, incluyendo Ecuador (22), lo cual ha condicionado la presencia de ofidios en el ambiente peridomiciliario, favoreciendo el contacto con el humano. Tanto la deforestación como las consecuencias del cambio climático son situaciones que podrían influenciar el aumento del ofidismo en el Paraguay.

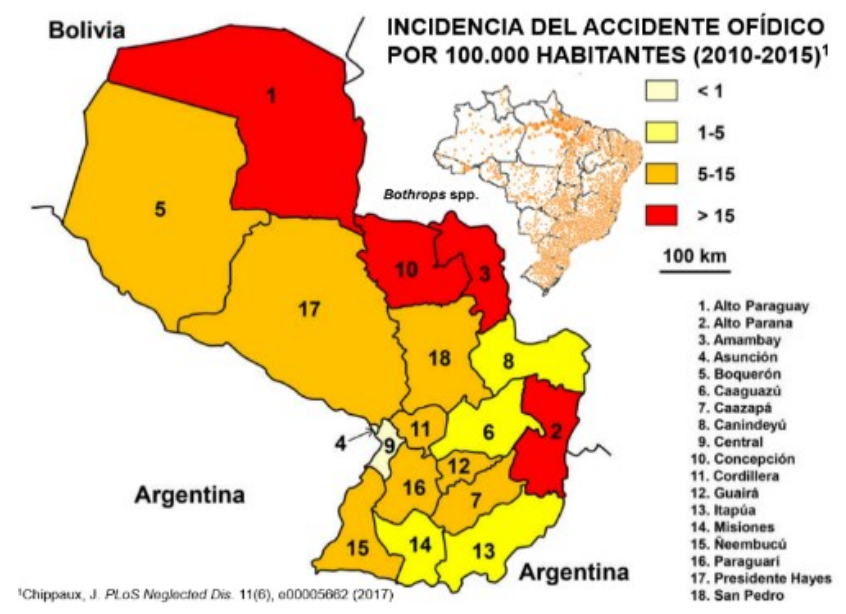

Figura 1: Incidencia del Accidente Ofídico por departamentos en el Paraguay (20102015) (modificado a partir de (4)).

\section{ESPECIES Y DISTRIBUCIÓN GEOGRÁFICA DE LAS SERPIENTES VIPÉRIDAS Y ELÁPIDAS DEL PARAGUAY}

La mayoría de los accidentes ofídicos en el Paraguay son debidos a serpientes pertenecientes a la familia Viperidae, la cual, junto con la familia Elapidae, contienen tres géneros y 14 especies de conocida o potencial importancia médica en el país (7) (Tabla 1). En la familia Viperidae, el género Bothrops (en comparación con Crotalus) es el de mayor relevancia sanitaria, con tres especies (de las seis reportadas para el Paraguay) identificadas como capaces de producir accidentes de elevada severidad: Bothrops alternatus (mbói kuatia), Bothrops jararaca (jarara) y Bothrops jararacusu (jararaguasu) (3).

Borges A. Ofidismo en el Paraguay: distribución geográfica, alteraciones fisiopatólogicas y necesidad de la preparación de un veneno de referencia nacional 
En el caso de la familia Elapidae, las serpientes de potencial importancia médica pertenecen al género Micrurus (mbói chumbe), con siete especies presentes en el Paraguay (7). La Figura 2 contiene fotografías representativas de las serpientes de los géneros Bothrops y Micrurus que habitan el país, junto a sus áreas de distribución geográfica.

El recuadro interno de la Figura 1 muestra la distribución de Bothrops. en el Brasil, indicando su potencial abundancia en la región oriental del Paraguay (8).

Tabla 1: Especies pertenecientes a las familias Viperidae y Elapidae de distribución en el Paraguay (basada en (7))

\begin{tabular}{ll} 
FAMILIA & GÉNERO/ ESPECIE \\
\hline VIPERIDAE & Bothrops alternatus \\
& Bothrops diporus \\
& Bothrops jararaca \\
& Bothrops jararacussu \\
& Bothrops moojeni \\
& Bothrops pauloensis \\
& Crotalus durissus \\
& Micrurus altirostris \\
& Micrurus ballocoryphus \\
& Micrurus corallinus \\
& Micrurus frontalis \\
& Micrurus silviae \\
& Micrurus lemniscatus \\
& Micrurus pyrrochriptus
\end{tabular}

\section{FISIOPATOLOGÍA DEL ENVENENAMIENTO OFÍDICO}

El complejo cuadro clínico que se desarrolla a raíz del envenenamiento ofídico es consecuencia de la acción de múltiples proteínas presentes en el veneno, cuya composición está sujeta a la edad de la serpiente, su dieta y origen geográfico, entre otras variables. Las manifestaciones clínicas locales más comunes observadas tras los accidentes por Bothrops spp. en Paraguay son edema y dolor, mientras que las complicaciones más evidentes son 
insuficiencia renal aguda, anemia, coagulación intravascular diseminada, necrosis, celulitis y abscesos, las cuales pueden ser explicadas en base a la fisiopatología del envenenamiento (10). La Figura 3 resume los principales mecanismos fisiopatológicos asociados al accidente ofídico, particularmente en el caso de serpientes pertenecientes a la familia Viperidae. La mayoría de los venenos de las serpientes vipéridas y algunas de la familia Elapidae inducen daño local extenso. La mionecrosis, o daño necrótico muscular, es principalmente debida a la presencia en estos venenos de fosfolipasas tipo A2 miotóxicas (PLA2s), las cuales son enzimas que destruyen la integridad de las membranas celulares debido a su acción sobre fosfolípidos. La entrada de calcio al citoplasma ocurre luego de la destrucción de la membrana, lo que causa hipercontracción de los miofilamentos y disfunción mitocondrial, entre otros eventos degenerativos, llevando al daño muscular irreversible. Las fibras musculares se afectan como resultado de la isquemia, producto de alteraciones vasculares y por la presión incrementada sobre el músculo a consecuencia del edema producido. La regeneración muscular requiere la remoción del tejido necrosado por parte de células fagocíticas, lo cual depende de un suministro sanguíneo intacto y de inervación para ser exitosa. En el caso de los venenos de serpientes vipéridas, los cuales afectan fibras musculares, vasos sanguíneos y nervios simultáneamente, la regeneración muscular está severamente limitada, resultando frecuentemente en secuelas de carácter irreversible. Adicionalmente a la mionecrosis, la integridad de los vasos sanguíneos es afectada, en vista de la presencia de metaloproteasas (SVMPs) que hidrolizan componentes claves de la membrana basal de los capilares, especialmente el colágeno tipo IV, debilitando la estabilidad mecánica de la microvasculatura. Como consecuencia, las fuerzas hemodinámicas biofísicas que operan en la circulación causan distensión y eventualmente la disrupción de la pared del capilar, resultando en extravasación. El daño microvascular inducido por las SVMPs también puede ser consecuencia de la ruptura de las uniones entre células endoteliales. Tanto las SVMPs como las hialuronidasas hidrolizan componentes de la matriz extracelular, incluyendo diferentes tipos de colágenos, ácido hialurónico y proteoglicanos, afectando la estructura y la función no sólo de la microvasculatura sino de otros componentes tisulares, jugando un papel central en el daño local tisular característico del envenenamiento por serpientes vipéridas, en particular las especies del género Bothrops. Las SVMPs también inducen daños en la piel producto de la degradación de la interfaz dermis-epidermis, con la consiguiente formación de ampollas. En consecuencia, se produce un proceso inflamatorio local en el tejido 
envenenado, con la síntesis de eicosanoides, óxido nítrico, bradicinina, histamina y citosinas, la activación de macrófagos residentes y el reclutamiento de linfocitos. Esta inflamación induce el aumento de la permeabilidad vascular con la formación de un exudado, que contiene fragmentos de proteínas extracelulares y citosinas pro-inflamatorias, el cual exacerba el proceso inflamatorio y aumenta el daño tisular (11).
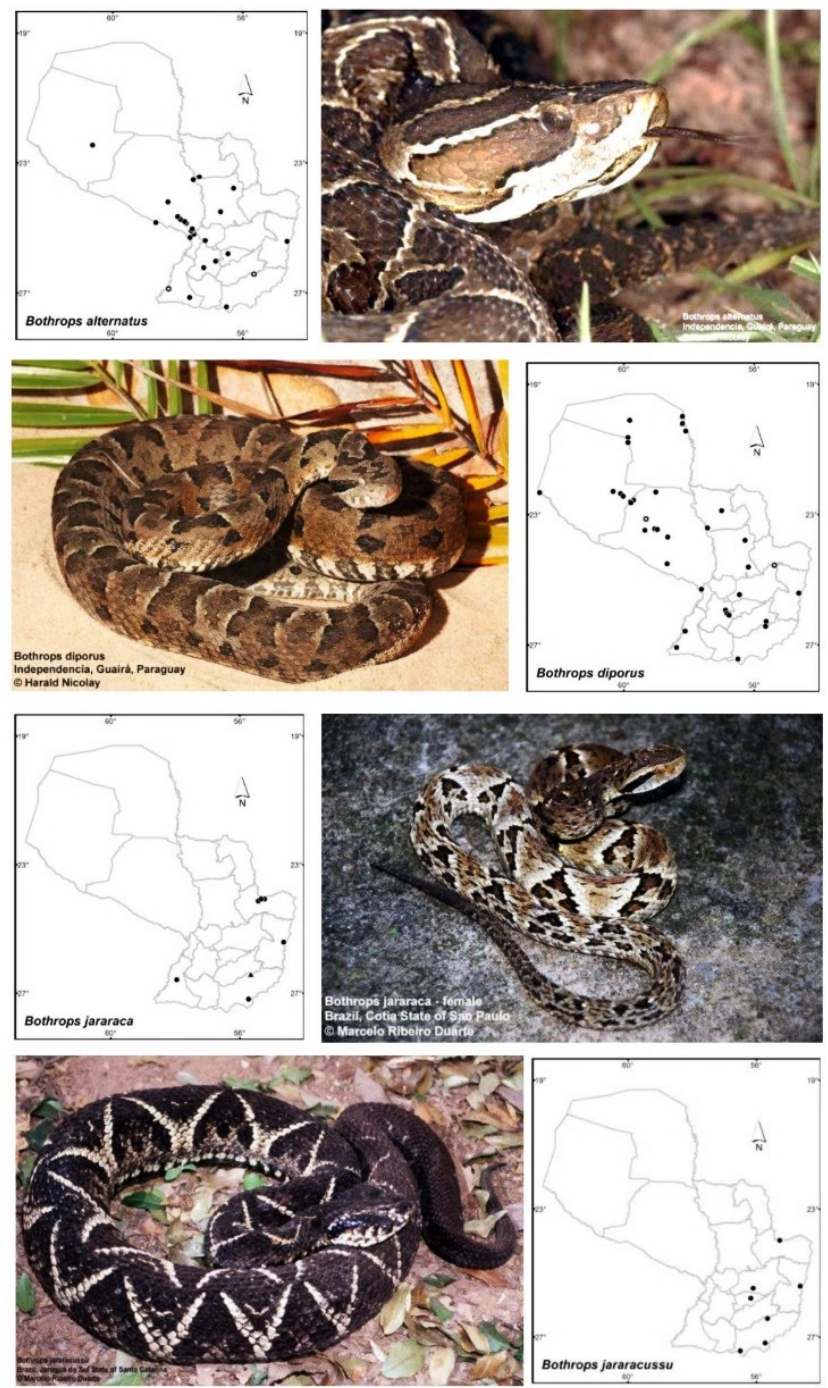

Borges A. Ofidismo en el Paraguay: distribución geográfica, alteraciones fisiopatólogicas y necesidad de la preparación de un veneno de referencia nacional 

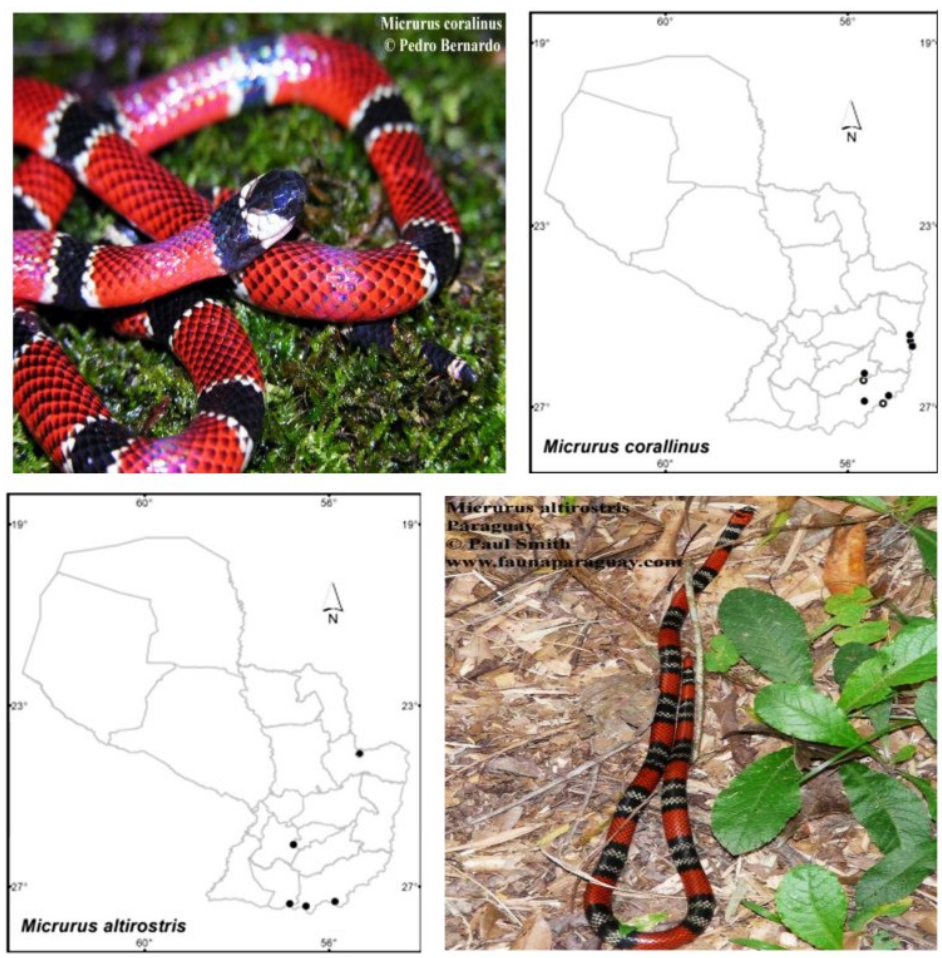

Figura 2: Serpientes de las famílias Viperidae (género Bothrops) y Elapidae (género Micrurus), distribución en el Paraguay.

En la Figura 2 se muestran las serpientes de las famílias Viperidae (género Bothrops) y Elapidae (género Micrurus) de distribución en el Paraguay, de conocida o potencial importancia médica. Las áreas de distribución geográfica, indicando los puntos en donde han sido colectadas las especies, fueron tomadas de (7). Las fotografías de los reptiles fueron tomadas de las siguientes fuentes: Bothrops alternatus (Independencia, Guairá, Paraguay) (fotografía por Harald Nicolay, http://reptiledatabase.reptarium.cz/); Bothrops diporus (Independencia, Guairá, Paraguay) (fotografía por Harald Nicolay, http://reptile-database.reptarium.cz/); Bothrops jararaca, estado de São Paulo, Brasil (fotografía por Marcelo Ribeiro Duarte, http://reptile-database.reptarium.cz/); Bothrops jararacussu, Santa Catarina, Brasil (fotografía por Marcelo Ribeiro Duarte, http://reptile-database.reptarium.cz/); Micrurus corallinus (fotografía por Pedro Bernardo, http://reptiledatabase.reptarium.cz/); Micrurus altirostris (fotografía por Paul Smith, http://reptile-database.reptarium.cz/). El lector es referido a (7) en el caso de la distribución de otras especies de Bothrops, Micrurus y Crotalus prevalentes en el Paraguay.

Borges A. Ofidismo en el Paraguay: distribución geográfica, alteraciones fisiopatólogicas y necesidad de la preparación de un veneno de referencia nacional 
Los venenos de las serpientes del género Bothrops afectan la hemostasia de diferentes maneras. La hemorragia sistémica inducida por el veneno es una de las causas principales de las alteraciones hemodinámicas que sufren estos pacientes, situación que puede progresar hacia un "shock" cardiovascular. Estos venenos contienen, además de SVMPs, enzimas tipo serina proteasas, que actúan sobre la cascada de coagulación, en la forma de enzimas parecidas a la trombina o como activadores del factor $\mathrm{V}$ de coagulación, el factor $\mathrm{X}$ o la protrombina. Algunas de las enzimas del veneno también hidrolizan fibrinógeno y fibrina. Adicionalmente, las SVMPs afectan la función endotelial de varias formas. Algunos de estos componentes procoagulantes pueden causar coagulación vascular diseminada, aunque en la mayoría de los casos inducen una coagulopatía por consumo, la cual resulta en pérdida del fibrinógeno e incoagulabilidad, lo cual se refleja en la alteración de los ensayos de coagulación (TP, TTP, fibrinógeno). Esta situación contribuye con la hemorragia sistémica que se produce en estos envenenamientos, especialmente en el caso de los venenos que contienen toxinas hemorrágicas que alteran la integridad de los vasos sanguíneos como es el caso de los venenos de Bothrops spp (11).

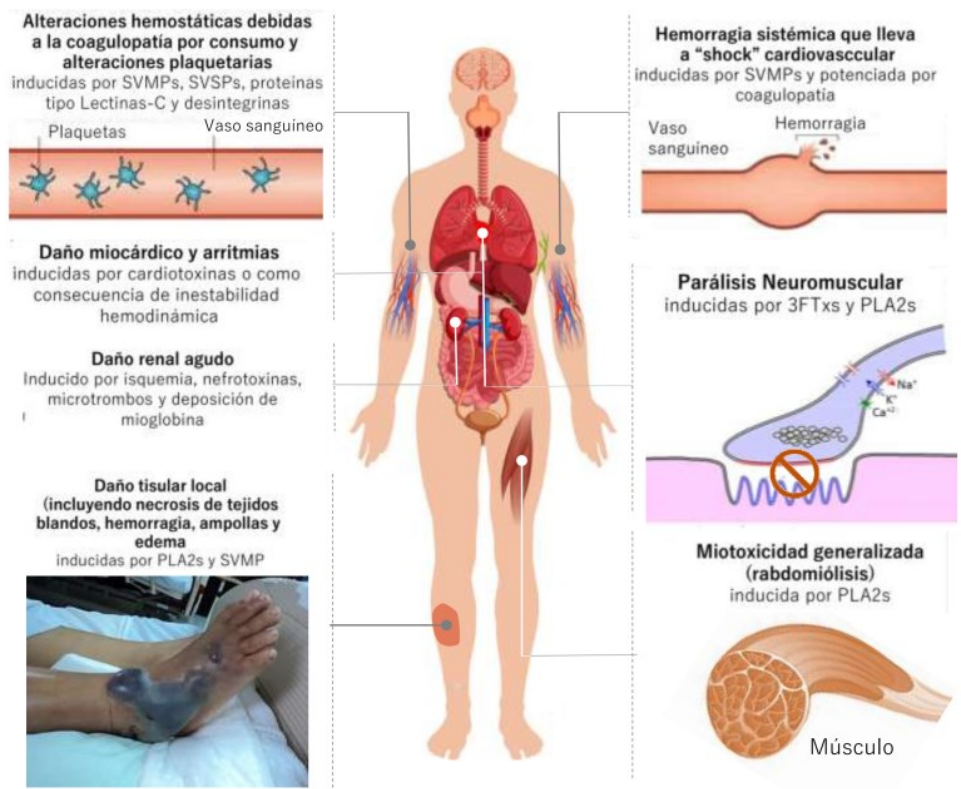

Figura 3: Mecanismos fisiopatológicos.

Borges A. Ofidismo en el Paraguay: distribución geográfica, alteraciones fisiopatólogicas y necesidad de la preparación de un veneno de referencia nacional 
En la Figura 3 se presentan los mecanismos fisiopatológicos asociados al envenenamiento por serpientes de las familias Viperidae y Elapidae (modificado a partir de (11)). SVMPs, metaloproteasas presentes en venenos de serpiente; SVSPs, serino proteasas presentes en venenos de serpientes; PLA2s, toxinas con actividad fosfolipásica. La fotografía del paciente mostrando ampollas a consecuencia del envenenamiento por Bothrops asper fue tomada por el Dr. Miguel Delgado, Hospital Luis Vernaza, Guayaquil, Ecuador.

En el caso de las serpientes pertenecientes a la familia Elapidae, incluyendo el género Micrurus, se presenta un síndrome clásico de neurotoxicidad, el cual está caracterizado por parálisis flácida, evidenciado al comienzo por ptosis bilateral y oftalmoplejia externa, acompañado en algunos casos de pupilas dilatadas. La parálisis desciende para involucrar músculos inervados por los nervios cranianos inferiores, así como los músculos flexores del cuello, bulbares, del tronco y las extremidades (11). Las toxinas presentes en estos venenos responsables de esta parálisis descendente que claramente compromete la vida del paciente, son las neurotoxinas $\alpha$ y $\beta$. Las primeras actúan post-sinápticamente a nivel de la unión neuromuscular. Se unen con gran afinidad a los receptores colinérgicos de la placa motora en fibras musculares, inhibiendo el enlazamiento de la acetilcolina y produciendo, en consecuencia, parálisis flácida. Por el contrario, las neurotoxinas $\beta$ son PLA2s que actúan a nivel presináptico de la unión neuromuscular. Luego de la unión a su receptor farmacológico, estas PLA2s inducen la hidrólisis de fosfolípidos a nivel de la membrana del terminal nervioso, lo cual causa neurotoxicidad. Adicionalmente, se incrementa la permeabilidad iónica de la membrana celular, produciéndose despolarización y entrada de calcio, lo que resulta en la exocitosis del "pool" de vesículas presinápticas. Ocurren, posteriormente, eventos degenerativos, incluyendo alteraciones mitocondriales, que llevan a la destrucción del terminal nervioso (12). Son necesarios estudios adicionales a fin de determinar la casuística debida a Micrurus spp. en el Paraguay y la posibilidad de que existan variaciones en la en la composición y la actividad biológica de los venenos de las poblaciones que habitan el país

\section{LA NECESIDAD DE PREPARAR ANTÍDOTOS ANTIOFÍDICOS PARA SU USO EN EL PARAGUAY}

El uso de antivenenos es la única terapia validada para el tratamiento del ofidismo a nivel global, por lo que estos medicamentos deben ser preparados con venenos de calidad trazable, siendo éstos además usados en los ensayos 
de control de calidad y preclínicos, con el fin de asegurar una adecuada eficacia (13). Paraguay depende de la importación de antivenenos para el tratamiento de estos accidentes $(2,14,15)$, por lo que, ante eventuales dificultades en el acceso a estos inmunobiológicos, es de importancia establecer un veneno de referencia nacional, que pudiese ser utilizado futuramente para la producción de antivenenos locales que neutralizaran la actividad tóxica específica de las poblaciones venenosas de Paraguay o, alternativamente, para el ensayo estandarizado de la capacidad neutralizante de nuevos antivenenos provenientes de otros países. De acuerdo a los lineamientos de la Organización Mundial de la Salud, un requisito indispensable para la producción de antivenenos es asegurar el uso de un veneno estándar científicamente caracterizado, tanto biológica como bioquímicamente, que sea representativo de las poblaciones de importancia médica prevalentes en el área geográfica de uso del antídoto, mismo que servirá, además de la producción, para el control de calidad, los estudios preclínicos y como referencia nacional para otros productores y para los trabajos de investigación que se generen (16). En este sentido, sería de utilidad la generación de tal veneno de referencia nacional de ofidios del Paraguay, cuya constitución debe estar basada en el estudio de las variaciones geográficas en composición y actividad tóxica de las poblaciones locales y de su importancia epidemiológica. Aunque han sido publicados varios estudios sobre la actividad biológica de venenos de especies de ofidios compartidas con Paraguay (17-19), es escasa la información sobre la variabilidad geográfica en composición y en actividad biológica de los venenos en el país y sobre la neutralización de su toxicidad por antídotos comerciales. Sólo está disponible en la literatura un reporte que indica que la dosis de antiveneno antibothrópico (sin especificar su origen) necesaria para neutralizar los efectos del envenenamiento por Bothrops spp. en Paraguay es de $150 \mathrm{mg}$ en la mayoría de los pacientes atendidos en el Instituto de Medicina Tropical de Asunción (174 pacientes internados desde octubre de 2005 a julio de 2007), si bien fue considerable el número de víctimas $(35,9 \%)$ que requirieron de dosis superiores del antídoto (desde 150 hasta $900 \mathrm{mg}$ ) (15). Para determinar la composición de dicho veneno de referencia, deben ser evaluados la reactividad antigénica de los venenos de las poblaciones de serpientes locales hacia los antivenenos foráneos disponibles en Paraguay y el grado de neutralización de las actividades tóxicas de dichos venenos por tales antídotos. 


\section{PERSPECTIVAS FUTURAS}

El ofidismo, al igual que el escorpionismo y el araneismo (20), son patologías que revisten gravedad en el Paraguay y cuya distribución temporal y espacial necesita ser mapeada combinando información sobre la historia natural de las especies venenosas con datos demográficos y epidemiológicos obtenidos a partir de las comunidades afectadas. El establecimiento de tales mapas de riesgo permitirá: (1) el diseño de estrategias de control que conduzcan a la reducción de la incidencia, (2) la optimización en la producción y distribución de antivenenos, y (3) el entrenamiento correcto del personal médico que trata al paciente envenenado. En este sentido, el estudio multidisciplinario de estas problemáticas por especialistas en Zoología, Epidemiología, Sistemas de Información Geográfica, Medicina Clínica, Bioquímica e Inmunología permitirá futuramente la comprensión y el abordaje holístico de estas patologías en el Paraguay.

\section{AGRADECIMIENTOS}

El autor agradece al Consejo Nacional de Ciencia y Tecnología (CONACyT) del Paraguay por el financiamiento otorgado a partir de la propuesta PRID18-12 en el marco del Programa de Radicación y Repatriación. Igualmente agradece al Programa Nacional de Incentivo al Investigador (PRONII-CONACyT). Al Dr. Miguel Delgado, por su gentileza al permitir la reproducción de la fotografía mostrada en la Figura 3.

\section{REFERENCIAS BIBLIOGRÁFICAS}

1. Vera A, Páez M, Gamarra de Cáceres G. Caracterización Epidemiológica de los accidentes ofídicos, Paraguay 2004, Mem. Inst. Investig. Cienc. Salud. 2006;4:20-24.

2. Gutiérrez JM, Fan HW, Silvera CL, Angulo Y. Stability, distribution and use of antivenoms for snakebite envenomation in Latin America: report of a workshop. Toxicon. 2009;53:625-630.

3. Alonso A, Vera R, Silva EO, Portillo C, Miret J, Canese J, Ramos P. Accidentes ofídicos notificados al Programa Nacional de Control de Zoonosis y Centro Antirrábico Nacional, Paraguay (2015). Rev. Salud Pública Parag. 2018;8(2):40-44.

4. Chippaux JP. Incidence and mortality due to snakebite in the Americas, PLoS Negl Trop Dis. 2017;11:e0005662.

5. Dirección General de Vigilancia de la Salud. Guía Nacional de Vigilancia y Control de Enfermedades. Asunción: Ministerio de Salud Pública y Bienestar Social, 2015. 
6. Hansson E, Sasa M, Mattisson K, Robles A, Gutiérrez JM. Using Geographical Information Systems to Identify Populations in Need of Improved Accessibility to Antivenom Treatment for Snakebite Envenoming in Costa Rica. PLoS Neglected Tropical Diseases. 2013;7:e2009.

7. Cacciali P, Scott NJ, Aquino Ortiz AL, Fitzgerald LA, Smith P. The Reptiles of Paraguay: Literature, Distribution, and an Annotated Taxonomic Checklist. Special Publication of The Museum of Southwestern Biology. 2016;11:1-373.

8. Yañez-Arenas C, Townsend Peterson A, Rodríguez-Medina K, Barve N. Mapping current and future potential snakebite risk in the new world. Climatic Change. 2016;134:697-711.

9. Nori J, Carrasco PA, Leynaud GC. Venomous snakes and climate change: ophidism as a dynamic problem. Climatic Change. 2013;122:67-80.

10. Moreno C. Epidemiología clínica y laboratorial por mordeduras de serpientes en pacientes hospitalizados. Rev. Inst. Med. Trop. (Asunción). 2011;6:23-27.

11. Gutiérrez JM, Calvete JJ, Habib AG, Harrison RA, Williams DJ, Warrell DA. Snakebite envenoming. Nat Rev Dis Primers. 2017;3:17063.

12. Rossetto 0, Montecucco C. Presynaptic neurotoxins with enzymatic activities. Handb. Exp. Pharmacol. 2008;184:129-170.

13. Williams DJ, Habib AG, Warrell DA. Clinical studies of the effectiveness and safety of antivenoms. Toxicon. 2018;150:1-10.

14. Ministerio de Salud Pública y Bienestar Social. Manual de Procedimientos sobre Accidentes Ofídicos. Asunción, Paraguay: MSPyBS, 2008.

15. Vicenti C, Taboada A, Aguilar G, Lezcano V, Alarcón R, Benítez G, Kunzle C. Dosis de suero antiofídico (SAO) necesaria para controlar los efectos tóxicos del veneno y la frecuencia de complicaciones. Asunción: Instituto de Medicina Tropical, 2007.

16. WHO. Blood Products and Related Biologicals. Geneva: WHO, 2010.

17. Roodt AR de, Estévez J, Dolab JA, Manzanelli MV, PiñeiroN, Paniagua JF, Vogt AU. Biological and immunological characteristics of the poison of Bothrops cotiara (Serpentes: Viperidae). Rev Biol Trop. 2006;54:889-901.

18. Cardoso KC, Da Silva MJ, Costa GG, Torres TT, Del Bem LE, Vidal RO, Menossi M, Hyslop S. A transcriptomic analysis of gene expression in the venom gland of the snake Bothrops alternatus (urutu). BMC Genomics. 2010;11:605.

19. Sandoval MP, Puorto G, Theakston RD, Warrell DA. Snake bites by the jararacuçu (Bothrops jararacussu): clinicopathological studies of 29 proven cases in São Paulo State, Brazil. QJM. 1997;90:323-334.

20. Borges A, Rojas de Arias a. El Accidente por Escorpiones Tóxicos en el Paraguay: Mito y Realidad en el contexto de la Emergencia por 
Escorpionismo en el Sudeste de la América del Sur. Revista de la Sociedad Científica del Paraguay. 2019;24:27-35.

21.Vera Sanabria MB, Ríos-González CM. Características clínicas y epidemiológicas de los accidentes ofídicos de un Hospital Regional de Paraguay, 2010 a 2016. Rev. Inst. Med. Trop. (Asunción). 2018;13:(2018) 21-30.

22. Boada C, Salazar D, Freire A, Kuch U. The diet of Bothrops asper (Garman, 1884 ) in the Pacific lowlands of Ecuador. Herpetozoa. 2005;18:77-79. 